\title{
Feasibility of improving platelet-rich plasma therapy by using chitosan with high platelet activation ability
}

\author{
HIDEMI HATTORI $^{1,2}$ and MASAYUKI ISHIHARA ${ }^{1}$ \\ ${ }^{1}$ Division of Biomedical Engineering, Research Institute, National Defense Medical College, \\ Tokorozawa, Saitama 359-8513; ${ }^{2}$ Department of Biochemistry and Applied Biosciences, Faculty \\ of Agriculture, University of Miyazaki, Miyazaki, Miyazaki 889-2192, Japan
}

Received July 21, 2015; Accepted October 28, 2016

DOI: 10.3892/etm.2017.4041

\begin{abstract}
Platelet-rich plasma (PRP) is blood plasma containing a high number of platelets that release growth factors for wound healing and tissue regeneration. In the present study, the feasibility of improving PRP therapy by using chitosan that exhibits high platelet activation ability was investigated. A total of 13 chitosan samples with different molecular weight $(\mathrm{Mw})$ and degree of deacetylation (DDA) were individually added to blood samples of rats and the amount of growth factors, albumin and fibrinogen in plasma was measured. To examine the influence of plasma activated by chitosan on the proliferation of fibroblasts and adipose tissue-derived stromal cells (ASCs), the plasma was added to the culture medium of human fibroblasts and adipose tissue-derived stromal cells. Chitosan with a DDA of $>75 \%$ increased the release of platelet factor 4 into the plasma. The amount of growth factors released into the plasma and platelet activation varied depending on the Mw and DDA, while albumin and fibrinogen were hardly affected. The proliferation rate was highest when using plasma activated by chitosan with a DDA of $75-85 \%$ and an Mw of 50,000-190,000 Da. These results suggested that the effectiveness of PRP therapy may be improved by using chitosan with a DDA of 75-85\% and an Mw of 50,000-190,000 Da.
\end{abstract}

\section{Introduction}

Platelet-rich plasma (PRP) is blood plasma with a high number of platelets. As an enriched source of autologous platelets, PRP contains several growth factors that are important for initiating and accelerating tissue repair and regeneration. Given these advantages, PRP therapy has

Correspondence to: Dr Hidemi Hattori, Department of Biochemistry and Applied Biosciences, Faculty of Agriculture, University of Miyazaki, 1-1 Gakuenkibanadai-nishi, Miyazaki, Miyazaki 889-2192, Japan

E-mail: h-hattori@cc.miyazaki-u.ac.jp

Key words: chitosan, platelet-rich plasma, growth factor, cell proliferation, platelet activation, deacetylation recently emerged as an innovative technique with great potential for healing chronic and acute wounds, including diabetic wounds, bedsores, skin ulcers and thermal burns. Fundamentally, the mechanisms underlying PRP therapy are the molecular and cellular stimulation of normal wound healing responses, similar to those observed during platelet activation (1). However, it is difficult to cure intractable diseases such as diabetic ulcers and decubitus, since the therapeutic effect tend to differ among individuals $(2,3)$. Reviving a wound with impaired healing is unmanageable because standard wound healing methods do not always provide improved healing results. This often demands more advanced therapies (4-6). Platelets can be activated upon two different types of stimuli: Physical stimuli, including heat, cold and vibration, and chemical stimuli, including collagen, lipopolysaccharide and chitosan (7-11). The activated platelets release biologically active substances and growth factors, such as platelet factor 4 (PF4), von Willebrand factor, platelet-derived growth factor (PDGF), hepatocyte growth factor (HGF), insulin-like growth factor (IGF) and vascular endothelial growth factor (12-14).

Chitosan is a polysaccharide derived from chitin, which is a compound of natural origin obtained from the shell of crabs and shellfish. Chitosan prepared from alkaline $\mathrm{N}$-deacetylation is composed of $\beta$-(1-4)-linked D-glucosamine and $N$-acetyl-D-glucosamine, which are randomly distributed. It carries a positive charge, as the free amino groups of $\beta$-(1-4)-linked D-glucosamine are protonated at physiological $\mathrm{pH}$. Chitosan is being extensively used as a potential biomaterial in several medical devices and health care products owing to its biodegradability and advantageous biological properties, including hemostatic activity $(15,16)$, biodegradability $(15,17)$, antibacterial activity $(18,19)$ and its ability to serve as a wound dressing agent to accelerate wound healing $(16,20,21)$. Given these advantages, the use of chitosan as a stimulant for platelet activation can be highly effective in PRP therapy. However, depending on the methods adopted for purifying chitosan from chitin, the molecular weight (Mw) and degree of deacetylation (DDA) vary. In other words, the function, properties and performance of chitosan are associated with their DDA and Mw.

Given these considerations, the present study proposed the concept of effective PRP therapy using chitosan. This strategy relies on the fact that chitosan activates platelets and enhances 
the release of growth factors into the plasma. To further enhance the effectiveness of PRP, a basic study using 13 different types of chitosan with varying Mw and DDA was performed.

\section{Materials and methods}

Animals and chitosan. The present study was approved by the Ethics Committee of Animal Care and Use, National Defense Medical College (Saitama, Japan) on July 28, 2014 (approval no., 14040) and the protocol was in accordance with the committee's guidelines for the care of animal subjects. Male Sprague-Dawley rats (28-48 weeks old; weight, 500-700 g; n=4) were obtained from Japan SLC (Hamamatsu, Japan). Following anesthesia with 3\% sevoflurane (Maruishi Pharmaceutical Co., Ltd., Osaka, Japan) inhalation, each $15-\mathrm{ml}$ blood sample collected from the tail vein was mixed with $3.13 \%$ sodium citrate solution $(10 \% \mathrm{v} / \mathrm{v})$ to inhibit coagulation. The blood sample was used for the examination as soon as it was collected. The Mw and DDA of each chitosan sample are listed in Table I. The samples with a DDA (in \%)/ Mw (in Da) of 84.2/8,600, 85.7/15,900, 50.3/28,800, 48.7/57,700, 35.5/30,000 and 33.6/57,300 (Yaizu Suisankagaku Industry Co., Ltd, Tokyo, Japan) were purified according to a previously described method (22). Chitosan oligomers (dimer, trimer, tetramer, pentamer and hexamer) were purchased from Seikagaku Co. (Tokyo, Japan), chitosan with a DDA of $87.6 \%$ and a Mw of 247,000 Da was obtained from Primex ehf (Siglufjordur, Iceland) and chitosan with a DDA of $75-85 \%$ and a Mw of 50,000-190,000 Da was from Sigma-Aldrich (Merck Millipore, Darmstadt, Germany). As an adjustment for the chitosan solution, $40 \mathrm{mg}$ of each chitosan powder was dissolved in $15 \mathrm{ml} 2 \%$ acetic acid, the $\mathrm{pH}$ was adjusted to 4.0 with $1 \mathrm{M}$ sodium bicarbonate, $2 \mathrm{ml}$ of $10 \mathrm{X}$ concentrated Dulbecco's phosphate-buffered saline (PBS) without calcium and magnesium was added to adjust to the osmotic pressure of blood, and the solution was topped up to $20 \mathrm{ml}$ with distilled water. Blank sample was adjusted as follows: $15 \mathrm{ml} 2 \%$ acetic acid was adjusted to $\mathrm{pH} 4.0$ with $1 \mathrm{M}$ sodium bicarbonate, $2 \mathrm{ml}$ of $10 \mathrm{X}$ concentrated PBS without calcium and magnesium was added and the solution was topped up to $20 \mathrm{ml}$ with distilled water.

Determination of protein in the plasma. In a typical process, $500 \mu \mathrm{l}$ of blood was added to $500 \mu \mathrm{l}$ of $0.2 \%$ chitosan solution and $25 \mu \mathrm{l}$ of $200 \mathrm{mM}$ calcium chloride solution. The solution was gently mixed and incubated at room temperature for $1 \mathrm{~h}$. Subsequently, the mixture was centrifuged at $10,000 \times \mathrm{g}$ for 15 min and the plasma was collected. The plasma samples were used at once without freezing. The levels of albumin, fibrinogen, PF4, PDGF-AB, PDGF-BB, IGF and HGF in the plasma were measured using ELISA kits as follows: Rat Albumin ELISA kit (E-25AL) and Rat Fibrinogen ELISA kit (E-25FIB), both from Immunology Consultants Laboratory (Portland, OR, USA); ELISA kit for Platelet Factor 4 (SEA172Ra; USCN Life Science, Wuhan, China); Mouse/Rat PDGF-AB Quantikine ELISA kit (MHD00), Mouse/Rat PDGF-BB Quantikine ELISA kit (MBB00) and Mouse/Rat IGF-I Quantikine ELISA kit (MG100), all from R\&D Systems, Inc. (Minneapolis, MN, USA); and Rat HGF EIA (1Z81; Institute of Immunology, Tokyo, Japan).
Table I. Chitosan samples used in the present study.

\begin{tabular}{lcc}
\hline Number & $\begin{array}{c}\text { Degree of deacetylation } \\
(\%)\end{array}$ & $\begin{array}{c}\text { Molecular weight } \\
(\mathrm{Da})\end{array}$ \\
\hline 1 & 84.2 & 8,600 \\
2 & 85.7 & 15,900 \\
3 & $75-85$ & $50,000-190,000$ \\
4 & 87.6 & 247,000 \\
5 & 50.3 & 28,800 \\
6 & 48.7 & 57,700 \\
7 & 35.5 & 30,000 \\
8 & 33.6 & 57,300 \\
II (dimer) & 100 & 413.25 \\
III (trimer) & 100 & 610.87 \\
IV (tetramer) & 100 & 808.49 \\
V (pentamer) & 100 & $1,006.11$ \\
VI (hexamer) & 100 & $1,203.72$ \\
\hline
\end{tabular}

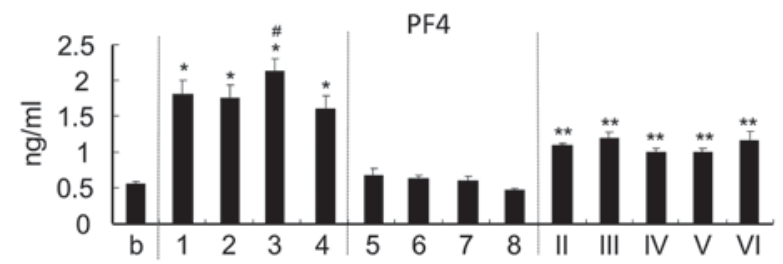

Figure 1. Amount of PF4 in plasma. Values are expressed as the mean \pm standard deviation $(\mathrm{n}=4)$. ${ }^{*} \mathrm{P}<0.0001,{ }^{* * *} \mathrm{P}<0.001$ vs. b. ${ }^{~} \mathrm{P}<0.05$ vs. all other samples. Each number corresponds to a chitosan sample defined in Table I. PF4, platelet factor 4; b, blank.

Cell proliferation assay. Plasma was sterilized using a $0.2-\mu \mathrm{m}$ filter (EMD Millipore, Billerica, MA, USA). Human fibroblasts (NHDF-Ad) and adipose tissue-derived stromal cells (ASCs; cat. no. PT-5006) (Lonza Japan, Tokyo) were plated at a density of $1.0 \times 10^{4}$ cells/well in 96-well culture plates (Sumitomo Bakelite Co., Ltd., Tokyo, Japan) and were cultured with Dulbecco's modified Eagle's medium including 5\% plasma. Cell proliferation was examined using a Cell Counting kit (Dojindo Co., Kumamoto, Japan).

Statistical analysis. Values are expressed as the mean \pm standard deviation. Multiple comparisons were evaluated using analysis of variance, as well as Tukey's and Dunnet's tests as appropriate. Statistical analysis was conducted using JMP ${ }^{\circledR}$ 11 software (SAS Institute Inc., Cary, NC, USA). P<0.05 was considered to indicate a statistically significant difference.

\section{Results}

Platelet activation. The platelet activation test was performed by measuring PF4 secretion in plasma. Chitosan with a high DDA $(>75 \%)$ including oligomer induced a higher release of PF4 in plasma than chitosan with a DDA of $50.3 \%$ and below (Fig. 1). In particular, chitosan with a Mw of 50,000-190,000 Da and a DDA of $75-85 \%$ exhibited the best activating efficiency among the 13 types of chitosan tested. 
A

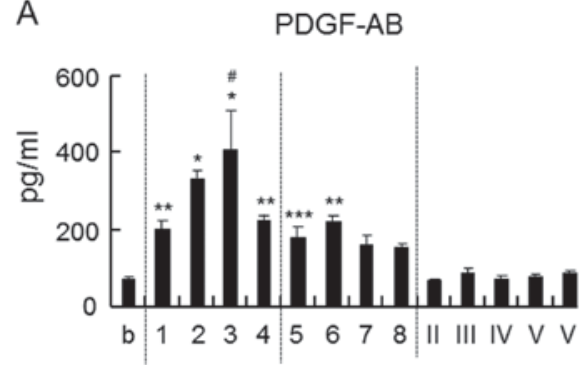

C

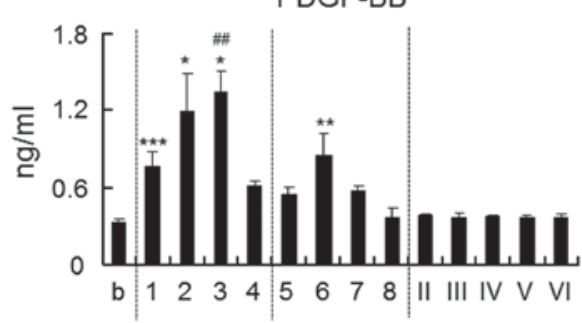

B

IGF-1

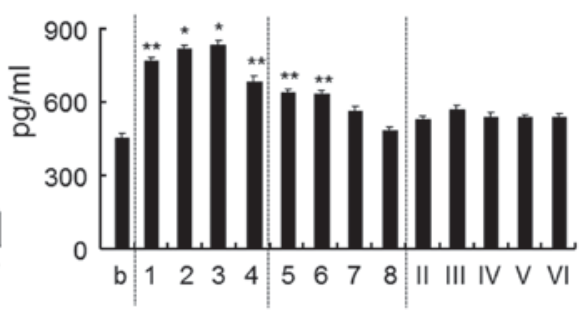

D

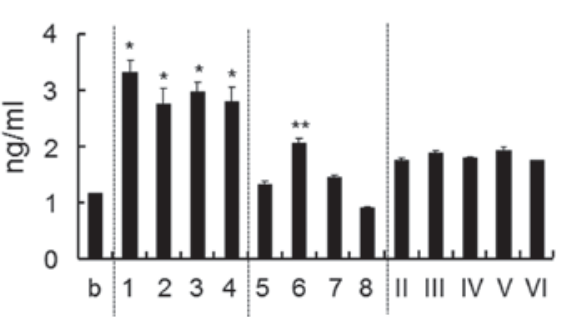

Figure 2. Amount of (A) PDGF-AB, (B) IGF-1, (C) PDGF-BB and (D) HGF in plasma. Each number corresponds to a chitosan sample defined in Table I. Values are expressed as the mean \pm standard deviation $(\mathrm{n}=4) .{ }^{*} \mathrm{P}<0.0001,{ }^{* *} \mathrm{P}<0.0005,{ }^{* * *} \mathrm{P}<0.001$ vs. $\mathrm{b} .{ }^{*} \mathrm{P}<0.0005,{ }^{\# \#} \mathrm{P}<0.05$ vs. all other samples. $\mathrm{PDGF}$, platelet-derived growth factor; IGF, insulin growth factor; HGF, hepatocyte growth factor; b, blank.
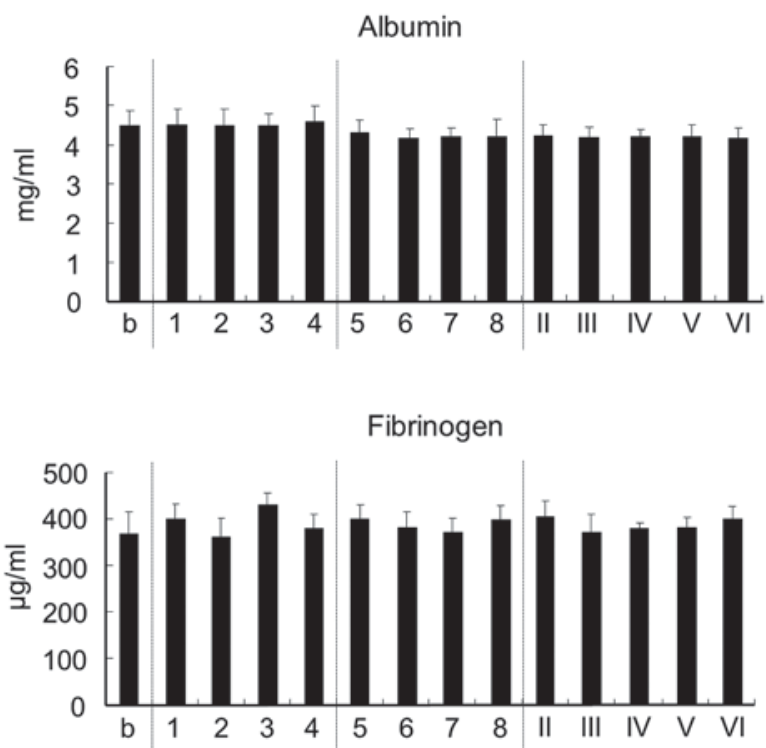

Figure 3. Amount of albumin and fibrinogen in plasma. Each number corresponds to a chitosan sample defined in Table I. Values are expressed as the mean \pm standard deviation $(n=4)$. b, blank.

Growth factors induced by chitosan. The amounts of growth factors such as PDGF-AB, PDGF-BB, IGF-1 and HGF in plasma were measured (Fig. 2). The amount of PDGF-AB and IGF-1 increased upon the addition of chitosan with a DDA of $>75 \%$ and a $\mathrm{Mw}$ of $>8,600 \mathrm{Da}$, as well as chitosan with a DDA of $\sim 50 \%$ and a Mw of 28,800 or 57,700 Da (Fig. 2A and B). The amount of PDGF-BB and HGF was increased upon addition of chitosan with a DDA of $>75 \%$ and a Mw of $>8,600 \mathrm{Da}$ as well as with a DDA of $48 \%$ and a Mw of 57,700 Da (Fig. 2C and D). Chitosan oligomer (100\% DDA) did not have any influence on the release of these growth factors.
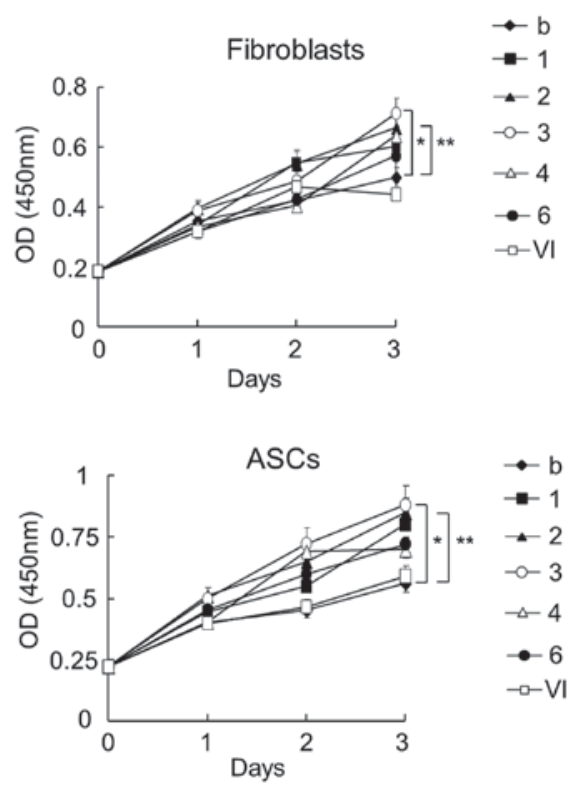

Figure 4. Proliferation of human fibroblasts and ASCs. Each number corresponds to a chitosan sample defined in Table I. Values are expressed as the mean \pm standard deviation $(n=4) .{ }^{*} \mathrm{P}<0.0005,{ }^{* *} \mathrm{P}<0.001$ vs. b. ASC, adipose tissue-derived stromal cell; OD, optical density; b, blank.

Effect on plasma protein. The effect of chitosan on plasma albumin, which accounts for $\sim 60 \%$ of all plasma protein, and on fibrinogen, which has an important role in secondary hemostasis, was measured. However, chitosan was found to have no effect on the levels of albumin and fibrinogen in plasma (Fig. 3).

Cell proliferation. To analyze the effect of chitosan-treated plasma on cell proliferation, human fibroblasts and ASCs were cultured using plasma activated by chitosan (Fig. 4). Based on the results of growth factors induced by chitosan (Fig. 2), 
the effects of plasma individually treated with 7 different chitosan samples on cell proliferation was examined. The use of plasma activated by chitosan with a DDA of $75-85 \%$ and a Mw of 50,000-190,000 Da resulted in the highest increase in the proliferation of fibroblasts and ASCs. The second-highest increase was achieved using chitosan with a DDA of $85.7 \%$ and a Mw of 15,900 Da. However, the chitosan oligomer did not increase the cell proliferation when compared to other chitosan samples.

\section{Discussion}

PRP therapy is a treatment process that uses a patient's own blood to activate and release of growth factor-rich granules at the wound site. Chitosan has been reported to improve the efficiency of PRP therapy (23-25). PRP-loaded chitosan scaffolds may be an appropriate carrier for PRP applications that facilitate the sustained release of growth factors (23). Chitosan is used as a functional delivery aid to simultaneously support PRP, stem cells and growth factors (24). Platelets contain negatively charged membranes due to the presence of negatively charged sugars, negatively charged phospholipids such as phosphatidylethanolamine phosphatidylcholine, carbohydrates such as sialic acid, and, to a much lesser extent, negatively charged amino acids such as aspartate and glutamate (26-29). By contrast, chitosan is positively charged due to the existence of free amino groups derived from the deacetylation of $\mathrm{N}$-acetyl-D-glucosamine. Chitosan is highly positively charged, and strongly attracts and binds to negatively charged molecules. These interactions potentially induce platelet activation. Normally, non-activated platelets store CD62P in the alpha-granule membranes, but several stimulators rapidly translocate to the platelet surface (30-33). Platelet activated by these stimulators releases alpha-granule constituents, such as PF4 and growth factors $(12,13)$. Although chitosan has previously been reported to cause platelet activation (34-37), the effect of differences in Mw and DDA has remained elusive. Therefore, basic studies using 13 types of chitosan with varying Mw and DDA were performed in the present study.

As it was assumed possible to produce more effective treatments for intractable diseases by use of PRP in which platelets are activated by chitosan, a preliminary experiment was performed to assess the platelet activation and the release of growth factors in PRP including chitosan; however, no accurate analysis was possible. Due to the platelet activation effect of chitosan, the PF4 levels and growth factors showed large variations due to being affected by slight stimulations occurring during the measurement procedure. Consequently, these factors were assayed in plasma, which was centrifuged after chitosan was added to the whole blood. Chitosan with a DDA of $>75 \%$, including chitosan oligomer, significantly enhanced PF4 release; chitosan with a reasonably high DDA therefore increased the platelet activation. This activation can be attributed to the increase in the number of free amino groups in chitosan with a higher DDA. A high DDA is therefore important for high platelet activation, and additionally, a Mw of >8,900 Da was also required for higher activation.

Platelets activated by chitosan released various growth factors, including PDGF-AB, PDGF-BB, HGF and IGF-1. However, the effects on the levels of growth factors were not the same among the different types of chitosan. The release of
PDGF-AB and PDGF-BB was the highest in the presence of chitosan with a DDA of 75-85\% and a Mw of 50,000-190,000 Da. However, this was not the case for IGF-1 and HGF, whose release was highest in the presence of chitosan with a DDA of $>75 \%$ and $\mathrm{Mw}$ of $8,600-190,000 \mathrm{Da}$ as well as with a DDA of $48 \%$ and a Mw of $57,700 \mathrm{Da}$. The observed difference in the levels of growth factors may possibly be attributed to the charge balance or interactions of other proteins. Further studies are required to explain for the high platelet activation observed in chitosan with a higher DDA and lower Mw. Overall the results of the present study demonstrated that the addition of chitosan to blood activates platelets to release growth factors in plasma, thereby improving the effectiveness of the PRP therapy.

Growth factors promote cell proliferation, differentiation and angiogenesis $(38,39)$. PRP induces stimulation of cell growth in ASCs, periodontal ligament and mesenchymal stem cells as well as enhancement of cellular adhesion, proliferation and differentiation of human periodontal ligament cells (40). In the present study, plasma with chitosan-induced growth factor enrichment stimulated the growth of fibroblasts and ASCs. In particular, the proliferation was enhanced with the use of plasma containing chitosan with a DDA of 75-85\% and a Mw of 50,000-190,000 Da. Recently, Bura et al (41) demonstrated the feasibility and safety of autologous ASC transplantation in patients with objectively proven critical limb ischemia not suitable for revascularization. The use of ASCs and PRP, which is activated by chitosan with a DDA of 75-85\% and a Mw of 50,000-190,000 Da for therapeutic application in wound healing and complications in patients with intractable diseases such as diabetic ulcers and decubitus, is expected to be an efficacious approach.

Fibrinogen is an acute-phase protein that is a part of the coagulation cascade, the end result of which is the production of thrombin that converts fibrinogen to fibrin clots. Surfaces of the materials coated with fibrinogen promote platelet adhesion and activation (42). Albumin, which accounts for $\sim 60 \%$ of plasma protein, is negatively charged, as are platelets (43). Albumin combines with various internal substrates and functions to transport them to the target tissue. In the present study, chitosan was not found to affect fibrinogen and albumin in plasma.

Of all the 13 chitosan samples tested in the present study, that with a DDA of $75-85 \%$ and a Mw of 50,000-190,000 Da showed the highest platelet activation and release of growth factors. Moreover, plasma induced by chitosan stimulated the proliferation of human fibroblasts and ASCs. However, chitosan did not affect the levels of fibrinogen and albumin in plasma. These results suggested that the effectiveness of PRP can be improved by using this type of chitosan.

\section{Acknowledgements}

The authors would like to thank Yaizu Suisankagaku Industry Co., Ltd (Tokyo, Japan) for supplying the chitosan, as well as Ms. Keiko Yamazaki and Ms. Reiko Yoshimoto for their research assistance.

\section{References}

1. Ferrari M,Zia S, Valbonesi M, Henriquet F, Venere G, Spagnolo S, Grasso MA and Panzani I: A new technique for hemodilution, preparation of autologous platelet-rich plasma and intraoperative blood salvage in cardiac surgery. Int J Artif Organs 10: 47-50, 1987. 
2. Greer N, Foman NA, MacDonald R, Dorrian J, Fitzgerald P, Rutks I and Wilt TJ: Advanced wound care therapies for nonhealing diabetic, venous, and arterial ulcers: A systematic review. Ann Intern Med 159: 532-542, 2013.

3. Jones KR, Fennie K and Lenihan A: Evidence-based management of chronic wounds. Adv Skin Wound Care 20: 591-600, 2007.

4. Carter MJ, Fylling CP and Parnell LK: Use of platelet rich plasma gel on wound healing: A systematic review and meta-analysis. Eplasty 11: e38, 2011

5. Steed DL, Attinger C, Colaizzi T, Crossland M, Franz M, Harkless L, Johnson A, Moosa H, Robson M, Serena T, et al: Guidelines for the treatment of diabetic ulcers. Wound Repair Regen 14: 680-692, 2006.

6. Bolton LL, van Rijswijk L and Shaffer FA: Quality wound care equals cost-effective wound care: A clinical model. Adv Wound Care 10: 33-38, 1997.

7. Zucker MB and Nachmias VT: Platelet activation. Arteriosclerosis 5: 2-18, 1985.

8. Winokur R and Hartwig JH: Mechanism of shape change in chilled human platelets. Blood 85: 1796-1804, 1995.

9. Kroll MH, Hellums JD, McIntire LV, Schafer AI and Moake JL: Platelets and shear stress. Blood 88: 1525-1541, 1996.

10. Brown GT, Narayanan P, Li W, Silverstein RL and McIntyre TM: Lipopolysaccharide stimulates platelets through an IL-1 $\beta$ autocrine loop. J Immunol 191: 5196-5203, 2013

11. Li Z, Delaney MK, O'Brien KA and Du X: Signaling during platelet adhesion and activation. Arterioscler Thromb Vasc Biol 30: 2341-2349, 2010.

12. Kowalska MA, Rauova L and Poncz M: Role of the platelet chemokine platelet factor 4 (PF4) in hemostasis and thrombosis. Thromb Res 125: 292-296, 2010.

13. Burnouf T, Goubran HA, Chen TM, Ou KL, El-Ekiaby M and Radosevic M: Blood-derived biomaterials and platelet growth factors in regenerative medicine. Blood Rev 27: 77-89, 2013.

14. Nakamura T, Teramoto $\mathrm{H}$ and Ichihara A: Purification and characterization of a growth factor from rat platelets for mature parenchymal hepatocytes in primary culture. Proc Natl Acad Sci USA 83: 6489-6493, 1986.

15. Hattori H, Amano Y, Nogami Y, Takase B and Ishihara M: Hemostasis for severe hemorrhage with photocrosslinkable chitosan hydrogel and calcium alginate. Ann Biomed Eng 38: 3724-3732, 2010.

16. Ono K, Ishihara M, Ozeki Y, Deguchi H, Sato M, Saito Y, Yura H, Sato M, Kikuchi M, Kurita A and Maehara T: Experimental evaluation of photocrosslinkable chitosan as a biologic adhesive with surgical applications. Surgery 130: 844-850, 2001.

17. Wedmore I, McManus JG, Pusateri AE and Holcomb JB: A special report on the chitosan-based hemostatic dressing: Experience in current combat operations. J Trauma 60: 655-658, 2006.

18. Sarasam AR, Brown P, Khajotia SS, Dmytryk JJ and Madihally SV: Antibacterial activity of chitosan-based matrices on oral pathogens. J Mater Sci Mater Med 19: 1083-1090, 2008.

19. Mellegard H, Kovács ÁT, Lindbäck T, Christensen BE, Kuipers OP and Granum PE: Transcriptional responses of Bacillus cereus towards challenges with the polysaccharide chitosan. PLoS One 6: e24304, 2011.

20. Shigemasa Y and Minami S: Applications of chitin and chitosan for biomaterials. Biotechnol Genet Eng Rev 13: 383-420, 1996.

21. Park CJ, Gabrielson NP, Pack DW, Jamison RD and Wagoner Johnson AJ: The effect of chitosan on the migration of neutrophil-like HL60 cells, mediated by IL-8. Biomaterials 30 : 436-444, 2009

22. Hattori $\mathrm{H}$ and Ishihara $\mathrm{M}$ : Changes in blood aggregation with differences in molecular weight and degree of deacetylation of chitosan. Biomed Mater 10: 015014, 2015.

23. Kutlu B, Tiğlı Aydın RS, Akman AC, Gümüşderelioglu M and Nohutcu RM: Platelet-rich plasma-loaded chitosan scaffolds: Preparation and growth factor release kinetics. J Biomed Mater Res B Appl Biomater 101: 28-35, 2013.
24. Busilacchi A, Gigante A, Mattioli-Belmonte M, Manzotti S and Muzzarelli RA: Chitosan stabilizes platelet growth factors and modulates stem cell differentiation toward tissue regeneration. Carbohydr Polym 98: 665-676, 2013

25. Oktay EO, Demiralp B, Demiralp B, Senel S, Cevdet Akman A, Eratalay $\mathrm{K}$ and Akincibay $\mathrm{H}$ : Effects of platelet-rich plasma and chitosan combination on bone regeneration in experimental rabbit cranial defects. J Oral Implantol 36: 175-184, 2010.

26. Bosmann HB: Platelet adhesiveness and aggregation: II. Surface sialic acid, glycoprotein: $\mathrm{N}$-acetylneuraminic acid transferase, and neuraminidase of human blood platelets. Biochim Biophys Acta 279: 456-474, 1972.

27. Lupu $\mathrm{C}$ and Calb M: Changes in the platelet surface charge in rabbits with experimental hypercholesterolemia. Atherosclerosis 72: 77-82, 1988 .

28. vd Winkel JG, Wetzels JF, van Duijnhoven JL, Koene RA and Capel PJ: Red blood cell surface charge and alcian blue binding. Nephrol Dial Transplant 2: 280-281, 1987.

29. Briedé JJ, Heemskerk JW, Hemker HC and Lindhout T: Heterogeneity in microparticle formation and exposure of anionic phospholipids at the plasma membrane of single adherent platelets. Biochim Biophys Acta 1451: 163-172, 1999.

30. Mackman N, Tilly RE and Key NS: Role of the extinsic pathway of blood coagulation in hemostasis and thrombosis. Arterioscler Thromb Vasc Biol 27: 1687-1693, 2007.

31. McEver RP: Adhesive interactions of leukocytes, platelets, and the vessel wall during hemostasis and inflammation. Thromb Haemost 86: 746-756, 2001.

32. Klinger $\mathrm{MH}$ : Platelets and inflammation. Anat Embryol (Berl) 196: 1-11, 1997.

33. Hagberg IA and Lyberg T: Evaluation of circulating platelet-leukocyte conjugates: A sensitive flow cytometric assay well suited for clinical studies. Platelets 11: 151-160, 2000.

34. Shen EC, Chou TC, Gau CH, Tu HP, Chen YT and Fu E: Releasing growth factors from activated human platelets after chitosan stimulation: A possible bio-material for platelet-rich plasma preparation. Clin Oral Implants Res 17: 572-578, 2006.

35. Fukusawa M, Abe $H$, Masaoka $T$, Orita $H$, Horikawa $H$, Campeau JD and Washio M: The hemostatic effect of deacetylated chitin membrane on peritoneal injury in rabbit model. Surg Today 22: 333-338, 1992.

36. International committee for standardization in hematology. Recommendation of measurement of erythrocyte sedimentation rate of human blood. Am J Clin Pathol 68: 505-507, 1977.

37. Sugamori T, Iwase H, Maeda M, Inoue $Y$ and Kurosawa H: Local hemostatic effects of microcrystalline partially deacetylated chitin hydrochloride. J Biomed Mater Res 49: 225-232, 2000.

38. Lubkowska A, Dolegowska B and Banfi G: Growth factor content in PRP and their applicability in medicine. J Biol Regul Homeost Agents 26 (2 Suppl 1): S3-S22, 2012.

39. Miyazawa K: Hepatocyte growth factor activator (HGFA): A serine protease that links tissue injury to activation of hepatocyte growth factor. FEBS 277: 2208-2214, 2010

40. Han J, Meng HX, Tang JM, Li SL, Tang Y and Chen ZB: The effect of different platelet-rich plasma concentrations on proliferation and differentiation of human periodontal ligament cells in vitro. Cell Prolif 40: 241-252, 2007.

41. Bura A, Planat-Benard V, Bourin P, Silvestre JS, Gross F, Grolleau JL, Saint-Lebese B, Peyrafitte JA, Fleury S, Gadelorge M, et al: Phase I trial: The use of autologous cultured adipose-derived stroma/stem cells to treat patients with non-revascularizable critical limb ischemia. Cytotherapy 16: 245-257, 2014.

42. He Q, Ao Q, Gong K, Zhang L, Hu M, Gong Y and Zhang X Preparation and characterization of chitosan-heparin composite matrices for blood contacting tissue engineering. Biomed Mater 5: 055001, 2010.

43. Busher JT: Serum albumin and globulin. In: Clinical Methods: The History, Physical and Laboratory Examinations. Walker KH, Hall DW and Hurst WJ (eds). 3rd edition. Butterworth Publishers, Boston, pp497-499, 1990. 